\title{
Understanding Users in Context: An In-Depth Introduction to Fieldwork for User Centered Design
}

\author{
Susan M. Dray and David A. Siegel \\ Dray \& Associates, Inc., 2007 Kenwood Parkway, Minneapolis, Minnesota 55405 USA \\ firstname. lastname@dray.com
}

\begin{abstract}
There is increased awareness of the need for design to be driven by deep understanding of users, their activity patterns, processes, needs and external influences--understanding that can only be gained by studying user behavior in the user's context. This requires understanding of how to plan and carry out observational studies of users, which is a new skill for many. In addition, fieldwork is bigger than any one methodology. Therefore, in this tutorial, we will take a fresh and deeper look at fundamental principles, teach a range of techniques, and examine important issues on which methods differ.
\end{abstract}

Keywords: Fieldwork, ethnography, user research, contextual inquiry, artifact walkthough, naturalistic usability evaluation.

\section{Introduction}

Observational research differs from other ways of gathering user data and complements other user-centered design (UCD) methods. For instance, a deep understanding of challenges in users' work patterns can help identify user requirements for new tools, or may spark entirely new product concepts. Information about the context of use that is gained from these studies can inform specific design decisions about a wide variety of things including navigation requirements and affordances of new products. In addition, the usage context and dynamics of use can contribute to building of robust scenarios both to design to and to test against.

Doing useful and valid field research is not as simple as just watching users, or talking with them in their environment. It requires ways to approach the inherent challenges of such research, such as dealing with the inherent ambiguity of qualitative data from field studies; risks of anecdotal evidence; balancing observation and inquiry; sampling bias; reducing the risks of premature closure, identifying and controlling (to the extent possible) reactive effects and demand characteristics.

Planning the field research is a critical first step in assuring its validity. Establishing a focus and creating a focus structure are crucial steps in preparing for field research. The focus determines what will be salient for the researcher, and helps guide the researcher in prioritizing the many avenues that can be pursued during the data gathering in the field. Defining the focus is probably the most critical step in determining how you set up the study and whether you will actually bring back useful and relevant information. 


\section{Three Data Gathering Techniques}

\subsection{Contextual Inquiry}

Contextual Inquiry depends upon interaction with users in real time, combined with observation of them as they do their tasks, as well as observation of their context. Unlike a conventional interview, contextual inquiry is very non-linear. Opportunities to probe deeply as well as opportunities to broaden the exploration arise unpredictably. Specific inquiry techniques help the researcher not only to elicit samples of behavior and explore contextual dependencies, but also to balance depth with breadth.

\subsection{Artifact Walkthrough}

The actual interview techniques of Artifact Walkthrough can resemble those of Contextual Inquiry. However, Artifact Walkthroughs apply these techniques in situations where it is difficult to observe the process of interest. For example, the process may be intermittent and difficult to capture in real time, or it might be inappropriate or impossible to interrupt it. Artifact Walkthroughs allow for exploration of these processes retrospectively while grounding the information in concrete evidence of behavior, which helps deal with the limitations of participant recall. They also provide openings into wider exploration of the user's process. As with actual behavioral observation, they often bring out aspects of the process that the researcher would not have thought to ask about and the user would have thought to mention

\subsection{Naturalistic Usability Evaluation}

Naturalistic usability evaluation encompasses a range of techniques for evaluating the user's interaction with technology in the user's natural context, based on the user's own goals and materials. It therefore focuses on tasks that are meaningful to each user or that incorporate the user's own content more easily than laboratory evaluations. They also allow you to study both usability and utility conjointly and to explore both discovery and task performance. 